Tôhoku Math. Journ.

39 (1987), 543-555.

\title{
NORMS OF HANKEL OPERATORS AND UNIFORM ALGEBRAS, II
}

\author{
TAKAHIKO NAKAZI*
}

(Received September 12, 1986)

\begin{abstract}
Let $H^{\infty}$ be an abstract Hardy space associated with a uniform algebra. Denoting by $(f)$ the coset in $\left(L^{\infty}\right)^{-1} /\left(H^{\infty}\right)^{-1}$ of an $f$ in $\left(L^{\infty}\right)^{-1}$, define $\|(f)\|=\inf \left\{\|g\|_{\infty}\left\|g^{-1}\right\|_{\infty} ; g \in(f)\right\}$ and $\gamma_{0}=\sup \left\{\|(f)\| ;(f) \in\left(L^{\infty}\right)^{-1} /\left(H^{\infty}\right)^{-1}\right\}$. If $\gamma_{0}$ is finite, we show that the norms of Hankel operators are equivalent to the dual norms of $H^{1}$ or the distances of the symbols of Hankel operators from $H^{\infty}$. If $H^{\infty}$ is the algebra of bounded analytic functions on a multiply connected domain, then we show that $\gamma_{0}$ is finite and we determine the essential norms of Hankel operators.
\end{abstract}

0. Introduction. Let $X$ be a compact Hausdorff space, let $C(X)$ be the algebra of complex-valued continuous functions on $X$, and let $A$ be a uniform algebra on $X$. For $\tau \in M_{A}$, the maximal ideal space of $A$, set $A_{0}=\{f \in A ; \tau(f)=0\}$. Let $m$ be a representing measure for $\tau$ on $X$.

The abstract Hardy space $H^{p}=H^{p}(m), 1 \leqq p \leqq \infty$, determined by $A$ is defined to be the closure of $A$ in $L^{p}=L^{p}(m)$ when $p$ is finite and to be the weak*-closure of $A$ in $L^{\infty}=L^{\infty}(m)$ when $p$ is infinite. Put $H_{0}^{p}=$ $\left\{f \in H^{p} ; \int_{X} f d m=0\right\}, K^{p}=\left\{f \in L^{p} ; \int_{X} f g d m=0\right.$ for all $\left.g \in A_{0}\right\}$ and $K_{0}^{p}=$ $\left\{f \in K^{p} ; \int_{X} f d m=0\right\}$. Then $H_{0}^{p} \subset K_{0}^{p}$ and $H^{p} \subset K^{p}$.

Let $Q^{(1)}$ be the orthogonal projection from $L^{2}$ to $\left(H^{2}\right)^{\perp}=\bar{K}_{0}^{2}$ and $Q^{(2)}$ the orthogonal projection from $L^{2}$ to $\bar{H}_{0}^{2}$. For a function $\phi$ in $L^{\infty}$ we denote by $M_{\phi}$ the multiplication operator on $L^{2}$ determined by $\phi$. As in the previous paper [14], two generalizations of the classical Hankel operators are defined as follows. For $\phi$ in $L^{\infty}$ and $f$ in $H^{2}$

$$
H_{\phi}^{(j)} f=Q^{(j)} M_{\phi} f \quad(j=1,2) \text {. }
$$

If $A$ is a disc algebra and $\tau(f)=\tilde{f}(0)$, where $\tilde{f}$ denotes the holomorphic extension of $f$ in $A$, then $\tau$ is in $M_{A}$. The normalized Lebesgue measure $m$ on the unit circle $T$ is a representing measure for $\tau$. Then $H^{2}$ is the classical Hardy space and $H_{0}^{2}=K_{0}^{2}$. Hence $H_{\phi}^{(1)}=H_{\phi}^{(2)}$ and it is the classical Hankel operator $H_{\phi}$. It is well known that

* This research was partially supported by Grant-in-Aid for Scientific Research, Ministry of Education. 
and

(a) $\left\|H_{\phi}\right\|=\left\|\phi+H^{\infty}\right\|$

(b) $\left\|H_{\phi}\right\|_{e}=\left\|\phi+H^{\infty}+C(T)\right\|$,

where the essential norm $\left\|H_{\phi}\right\|_{e}$ of $H_{\phi}$ is the distance to the compact operators. (a) is due to Nehari (cf. [16, Theorem 1.3], [15]), while (b) is due to Adamyan, Arov and Krein (cf. [16, p. 6], [2]). (b) yields Hartman's result (cf. [16, Theorem 1.4], [11]) to the effect that

(c) $H_{\phi}$ is compact if and only if $\phi$ is in $H^{\infty}+C(T)$.

In the previous paper [14] we considered the generalizations of (1). The main idea was to consider Hankel operators on $v H^{2}$ for every nonnegative invertible function $v$ in $L^{\infty}$, avoiding a factorization theorem of $H_{0}^{1}$. Namely, if $h$ is in $H_{0}^{1}$ and $\int_{X}|h| d m \leqq 1$, then $h=f g, f \in H^{2}$ and $g \in H_{0}^{2}$ where $\int_{X}|f|^{2} d m \leqq 1+\varepsilon$ and $\int_{X}|g|^{2} d m \leqq 1+\varepsilon$ for some $\varepsilon>0$. Let $v$ be a nonnegative function in $L^{\infty}$ with $v^{-1}$ in $L^{\infty}$. Let $Q_{v}^{(1)}$ be the orthogonal projection from $L^{2}$ onto $\left(v H^{2}\right)^{\perp}=v^{-1} \bar{K}_{0}^{2}$ and $Q_{v}^{(2)}$ the orthogonal projection from $L^{2}$ onto $v^{-1} \bar{H}_{0}^{2}$. If $v$ is a constant function, then $Q_{v}^{(j)}=Q^{(j)}(j=$ 1,2). For $\phi \in L^{\infty}$ and $f \in v H^{2}, H_{\phi}^{(j) v}$ is the operator defined by

$$
H_{\phi}^{(j) v} f=Q_{v}^{(j)} M_{\phi} f, \quad(j=1,2) .
$$

If $v$ is a nonzero constant, then $H_{\phi}^{(j) v}=H_{\phi}^{(j)}(j=1,2)$. Put $\left(L^{\infty}\right)_{+}^{-1}=$ $\left\{v \in L^{\infty}: v^{-1} \in L^{\infty}\right.$ and $\left.v \geqq 0\right\}$. The following theorem was shown in the previous paper [14] and gives (a).

Generalized Nehari's Theorem I. Let $\phi$ be a function in $L^{\infty}$, then

$$
\sup \left\{\left\|H_{\phi}^{(2) v}\right\| ; v \in\left(L^{\infty}\right)_{+}^{-1}\right\}=\left\|\phi+K^{\infty}\right\| \text {. }
$$

If $K^{\infty}$ is dense in $K^{1}$, then

$$
\sup \left\{\left\|H_{\phi}^{(1) v}\right\| ; v \in\left(L^{\infty}\right)_{+}^{-1}\right\}=\left\|\phi+H^{\infty}\right\| .
$$

We now show two lemmas which will be used in later sections. Let $P_{v}$ be the orthogonal projection from $L^{2}$ onto $v H^{2}$. If $v$ is a constant function, we shall write $P_{v}=P$.

LEMma 1. Let $\phi$ be a function in $L^{\infty}$. Then for any $v$ and $u$ in $\left(L^{\infty}\right)_{+}^{-1}$ and for $j=1,2$

$$
\left\|H_{\phi}^{(j) v}-H_{\phi}^{(j) u}\right\| \leqq\|\phi\|_{\infty}\left(\left\|v^{-1}\right\|_{\infty}+\left\|u^{-1}\right\|_{\infty}\right)\|v-u\|_{\infty} .
$$

Proof. Since $\left\|M_{v} f\right\|_{2} \geqq\left\|v^{-1}\right\|_{\infty}^{-1}\|f\|_{2}$ for all $f \in H^{2}$, by [7, Lemma 1.1.]

$$
\left\|P_{v}-P_{u}\right\| \leqq\left(\left\|v^{-1}\right\|_{\infty}+\left\|u^{-1}\right\|_{\infty}\right)\|v-u\|_{\infty} .
$$

Similarly, $\left\|Q_{v}^{(j)}-Q_{u}^{(j)}\right\| \leqq\left(\left\|v^{-1}\right\|_{\infty}+\left\|u^{-1}\right\|_{\infty}\right)\|v-u\|_{\infty}$. Hence for $j=1,2$ 


$$
\begin{aligned}
\left\|H_{\phi}^{(j) v}-H_{\phi}^{(j) u}\right\| & =\left\|Q_{v}^{(j)} M_{\phi} P_{v}-Q_{u}^{(j)} M_{\phi} P_{v}+Q_{u}^{(j)} M_{\phi} P_{v}-Q_{u}^{(j)} M_{\phi} P_{u}\right\| \\
& \leqq\left\|Q_{v}^{(j)}-Q_{u}^{(j)}\right\|\left\|M_{\phi} P_{v}\right\|+\left\|P_{v}-P_{u}\right\|\left\|Q_{u}^{(j)} M_{\phi}\right\| \\
& \leqq\|\phi\|_{\infty}\left(\left\|v^{-1}\right\|_{\infty}+\left\|u^{-1}\right\|_{\infty}\right)\|v-u\|_{\infty} \cdot
\end{aligned}
$$

LEMMA 2. Let $\phi$ be a function in $L^{\infty}$. If $H_{\phi}^{(j)}(j=1,2)$ is compact, then $H_{\phi}^{(j) v}(j=1,2)$ is compact for any $v$ in $\left(L^{\infty}\right)_{+}^{-1}$.

Proof. For any $f \in v H^{2}$ and $g \in v^{-1} \bar{K}_{0}^{2},\left(H_{\phi}^{(1)} v f, g\right)=\left(v H_{\phi}^{(1)}\left(v^{-1} f\right), g\right)$. Hence $H_{\phi}^{v} f=Q^{(1) v}\left(M_{v} H_{\phi}^{(1)} M_{v}^{-1} f\right)$ for any $f \in v H^{2}$. The proof for $H_{\phi}^{(2) v}$ is similar. This implies the lemma.

Let $N_{\tau}$ denote the set of representing measures for $\tau$ on $X$. In this paper we sometimes will impose the following two conditions on $\tau$ :

(1) $N_{\tau}$ is finite dimensional and $n=\operatorname{dim} N_{\tau}$.

(2) $m$ is a core measure of $N_{\tau}$.

Let $N^{\infty}$ be the real annihilator of $A$ in $L_{R}^{\infty}$. Then $\operatorname{dim} N^{\infty}=n$ and $A+$ $\bar{A}_{0}+N_{c}^{\infty}$ is weak ${ }^{*}$-dense in $L^{\infty}$, where $N_{c}^{\infty}=N^{\infty}+i N^{\infty}$ (cf. [10, p. 109]). $L^{2}=H^{2} \oplus \bar{H}_{0}^{2} \oplus N_{c}^{\infty}$. Set $\mathscr{E}=\exp N^{\infty}$; then $\mathscr{E}$ is a subgroup of $\left(L^{\infty}\right)_{+}^{-1}$. Moreover, together with (1) we often will make the following stronger conditions (3) on $\tau$ instead of (2).

(3) $m$ is a unique logmodular measure of $N_{\tau}$.

Then the linear span of $N^{\infty} \cap \log \left|\left(H^{\infty}\right)^{-1}\right|$ is $N^{\infty}$ (cf. [10, p. 114]). Choose $h_{1}, \cdots, h_{n} \in\left(H^{\infty}\right)^{-1}$ so that $\left\{\log \left|h_{j}\right|\right\}_{j=1}^{n}$ is a basis for $N^{\infty}$. Put $u_{j}=\log \left|h_{j}\right|$ $(1 \leqq j \leqq n)$ and $\mathscr{E}_{0}=\left\{\exp \left(\sum_{j=1}^{n} s_{j} u_{j}\right) ; 0 \leqq s_{j} \leqq 1\right\}$. Then $\mathscr{E}_{0} \subset \mathscr{E}$. The following theorem was shown in the previous paper [14].

Generalized Nehari's Theorem II. Assume the assumptions (1) and (3) on $\tau$. Let $\phi$ be a function in $L^{\infty}$, then

and

$$
\sup _{v \in \mathscr{E}_{0}}\left\|H_{\phi}^{(2) v}\right\|=\left\|\phi+H^{\infty}+N_{c}^{\infty}\right\|
$$

$$
\sup _{v \in \mathscr{E}_{0}}\left\|H_{\phi}^{(1) v}\right\|=\left\|\phi+H^{\infty}\right\|
$$

Moreover the supremums in both equalities are attained.

In Section 1, $\gamma_{0}$, which is defined in Abstract, is studied. Under the assumptions (1) and (2) we determine when $\gamma_{0}$ is finite. In Section 2, we give examples of concrete uniform algebras to which results in this paper can apply. Moreover $\gamma_{0}$ is calculated in some examples. In Section 3, if $\gamma_{0}$ is finite, we show that $\left\|H_{\phi}^{(1)}\right\|$ (resp. $\left\|H_{\phi}^{(2)}\right\|$ ) is equivalent to $\left\|\phi+H^{\infty}\right\|$ (resp. $\left.\left\|\phi+K^{\infty}\right\|\right)$. In Section 4 we give applications of results in Section 3 to weighted norm inequalities for conjugation operators and invertible Toeplitz operators in uniform algebras. In Section 5 we determine the 
essential norms of Hankel operators in the case of (I) in Section 2. In Section 6 we consider the relationship between $\gamma_{0}$ and the factorization theorem of $H_{0}^{1}$. In Section 7 we consider the relationship between generalized Nehari's Theorem and Arveson's distance formula for nest algebras.

1. Quotient group and a constant. Denoting by $(f)$ the coset in $\left(L^{\infty}\right)^{-1} /\left(H^{\infty}\right)^{-1}$ of an $f$ in $\left(L^{\infty}\right)^{-1}$, define

$$
\|(f)\|=\inf \left\{\|g\|_{\infty}\left\|g^{-1}\right\|_{\infty} ; g \in(f)\right\}
$$

and

$$
\gamma_{0}=\sup \left\{\|(f)\| ;(f) \in\left(L^{\infty}\right)^{-1} /\left(H^{\infty}\right)^{-1}\right\} .
$$

Then $\|(f)(h)\| \leqq\|(f)\|\|(h)\|$ and, in general, $\gamma_{0}$ can be finite or infinite. Let $L_{R}^{\infty}$ be the space of real-valued functions in $L^{\infty}$. Let $\log \left|\left(H^{\infty}\right)^{-1}\right|$ be the lattice in $L_{R}^{\infty}$ consisting of the elements of the form $\log |f|, f \in\left(H^{\infty}\right)^{-1}$. There is a natural map of $\left(L^{\infty}\right)^{-1} /\left(H^{\infty}\right)^{-1}$ onto $L_{R}^{\infty} / \log \left|\left(H^{\infty}\right)^{-1}\right|$ which sends $(f)$ to $(\log |f|)$. Define $\||(\log |f|)|\|=\inf \left\{\|\log |f|+\log |g|\|_{\infty} ; g \in\left(H^{\infty}\right)^{-1}\right\}$ and $\gamma_{1}=$ $\sup \left\{||(\log |f|)|| ; ;(\log |f|) \in L_{R}^{\infty} / \log \left|\left(H^{\infty}\right)^{-1}\right|\right\}$.

Proposition 1. $\|(f)\|=\exp 2||(\log |f|)|| \mid$ and $\gamma_{0}=\exp 2 \gamma_{1}$.

Proof. It suffices to show that $\|(f)\|=\exp 2 \mid\|(\log |f|)\| \|$ for all $f \in$ $\left(L^{\infty}\right)^{-1}$. Pick such an $f$.

$$
\begin{aligned}
\|(f)\| & =\inf \left\{\|f g\|_{\infty}\left\|f^{-1} g^{-1}\right\|_{\infty} ; g \in\left(H^{\infty}\right)^{-1}\right\} \\
& =\exp \inf \{\operatorname{ess} . \sup (\log |f|+\log |g|)-\text { ess. } \inf (\log |f|+\log |g|)\} .
\end{aligned}
$$

Since the constants are in $\left(H^{\infty}\right)^{-1}$, this last quantity can be rewritten as

$$
=\exp 2 \inf \{\text { ess. } \sup |\log | f|+\log | g||\}=\exp 2|||(\log |f|)||| .
$$

The proof of Proposition 1 is parallel to that of Proposition 2.2 in [17]. Rochberg [17] considered $\left(H^{\infty}\right)^{-1} / \operatorname{exp~} H^{\infty}$ instead of $\left(L^{\infty}\right)^{-1} /\left(H^{\infty}\right)^{-1}$. If $A$ is a disc algebra, then $\|(f)\|=1$ for any $(f) \in\left(L^{\infty}\right)^{-1} /\left(H^{\infty}\right)^{-1}$ and so $\gamma_{0}=1$ because of Proposition 1 and $L_{R}^{\infty}=\log \left|\left(H^{\infty}\right)^{-1}\right|$. Let crls $\log \left|\left(H^{\infty}\right)^{-1}\right|$ denote the closed real linear span of $\log \left|\left(H^{\infty}\right)^{-1}\right|$.

LEMMA 3. (1) If $v=\sum_{j=1}^{n} t_{j} \log \left|h_{j}\right|$ with $0 \leqq t_{j} \leqq 1$ and $h_{j} \in\left(H^{\infty}\right)^{-1}$ $(1 \leqq j \leqq n)$, then $\sup \{\|(t v)\| ;-\infty<t<\infty\}<\infty$. (2) If $v \in L_{R}^{\infty}$ is not in crls $\log \left|\left(H^{\infty}\right)^{-1}\right|$, then $\sup \{\|t v \mid\| ;-\infty<t<\infty\}=\infty$.

Proof. (1) $\|(t v)\|\left|=\left\|\left|\left(\sum_{j=1}^{n} t t_{j} \log \left|h_{j}\right|\right)\right|\right\|=\right|\left\|\left(\sum_{j=1}^{n}\left(t t_{j}-\left[t t_{j}\right]\right) \log \left|h_{j}\right|\right)\right\|$, where [.] is the greatest integer function. Hence $\sup \|(t v)\|<\infty$.

(2) There exists a positive constant $\varepsilon$ such that $\left\|t v+\operatorname{crls} \log \left|\left(H^{\infty}\right)^{-1}\right|\right\|_{\infty} \geqq$ $\varepsilon\|t v\|_{\infty}$ for any $t$. Hence $\sup \|(t v)\| \|=\infty$.

THEOREM 2. Suppose $\tau$ satisfies the conditions (1) and (2). Then 
$m$ is a unique logmodular measure if and only if $\gamma_{0}$ is finite.

Proof. By Proposition 1 it is sufficient to show that $m$ is a unique logmodular measure if and only if $\gamma_{1}$ is finite. $m$ is a unique logmodular measure if and only if crls $\log \left|\left(H^{\infty}\right)^{-1}\right|=L_{R}^{\infty}$ (see $[10$, p. 114]). By this and (2) in Lemma 3, if $m$ is not a unique logmodular measure, then $\gamma_{1}=\infty$. Suppose $m$ is a unique logmodular measure. If $v \in L_{R}^{\infty}$, then $v=u_{0}+\log |g|$ with $u_{0} \in N^{\infty}$ and $g \in\left(H^{\infty}\right)^{-1}$ (cf. [10, p. 109]). Moreover, we can choose $u_{0} \in \log \mathscr{E}_{0}$ (see Introduction). By (1) in Lemma $3 \gamma_{1}$ is finite and in fact $\gamma_{1} \leqq \sup \left\{\left\|\sum_{j=1}^{n} s_{j} u_{j}\right\|_{\infty} ; 0 \leqq s_{j} \leqq 1\right\}$

2. Concrete uniform algebras. (I) Let $Y$ be a compact subset of the plane, and let $R(Y)$ be the uniform closure of the set of rational functions in $C(Y)$. We regard $R(Y)$ as a uniform algebra on its Shilov boundary, the topological boundary $X$ of $Y$. Suppose the complement $Y^{c}$ of $Y$ has a finite number $n$ of components and the interior $Y^{0}$ of $Y$ is a nonempty connected set. Let $A=R(Y) \mid X$; then $M_{A}=Y$. If $\tau \in M_{A}$ is in $Y^{0}$ and $m$ is a harmonic measure, then $m$ is a unique logmodular measure of $N_{\tau}$ and $\operatorname{dim} N_{\tau}=n<\infty$ [10, p. 116]. Then $N^{\infty} \subset C(X)$ By Theorem 2, $\gamma_{0}$ is finite. Let $X=X_{0} \cup X_{1} \cup \cdots \cup X_{n}$, where $X_{0}$ is the "outside" component of $X$ and $X_{1}, \cdots, X_{n}$ are the "inside" components of $X$. Define $v_{j} \in L_{R}^{\infty}$ to be 1 on $X_{j}$ and 0 on $X \backslash X_{j} 1 \leqq j \leqq n$. Then $\gamma_{1}=$ $\sup \left\{\left\|\left|\left(\sum_{j=1}^{n} t_{j} v_{j}\right)\right|\right\| ;-\infty<t_{j}<\infty\right.$ and $\left.1 \leqq j \leqq n\right\}$.

(II) In (I) let $Y$ be the annulus $\{r \leqq|z| \leqq 1\}$. Then $\gamma_{0}=r^{-1 / 2}$. Since $\left(L_{R}^{\infty} /\right.$ the uniform closure of $\operatorname{Re} H^{\infty}$ ) has dimension one, we get

$\gamma_{1}=\sup _{0 \leqq t \leqq 1} \inf \left\{|| t \log |z|-(\operatorname{Re} f+n \log |z|) \|_{\infty} ; f \in H^{\infty}\right.$ and $n$ ranges over all integers $\}$.

For any integer $n$

$$
\begin{aligned}
& \inf \left\{\|(t-n) \log |z|-\operatorname{Re} f\|_{\infty} ; f \in H^{\infty}\right\} \\
& \quad=|t-n| \log r^{-1} \inf \left\{\left\|\chi_{E}-\operatorname{Re} f\right\|_{\infty} ; f \in H^{\infty}\right\}=\frac{1}{2}|t-n| \log r^{-1},
\end{aligned}
$$

where $\chi_{E}=0$ on $|z|=1$ and $\chi_{E}=1$ on $|z|=r$. Thus

$$
\gamma_{1}=\sup _{0 \leqq t \leqq 1} \inf _{n} \frac{1}{2}|t-n| \log r^{-1}=\frac{1}{4} \log r^{-1} \text {. }
$$

We shall show that

$$
\inf \left\{\left\|\chi_{E}-\operatorname{Re} f\right\|_{\infty} ; f \in H^{\infty}\right\}=1 / 2 .
$$

Choosing $f=1 / 2$, the infimum is not greater than $1 / 2$. If the infimum is less than $1 / 2$ then by a theorem of Runge we can show that $\chi_{E} \in H^{\infty}$ as in the proof of Theorem in [13, p. 182]. This contradiction implies 
that the infimum is just $1 / 2$.

(III) Let $\mathscr{A}$ be the disc algebra and let $A$ be a subalgebra of $\mathscr{A}$ which contains the constants and which has finite codimension in $\mathscr{A}$. If $\tau(f)=\tilde{f}(0)$ for $f \in A$ and $m$ is the normalized Lebesgue measure on the circle $T$, then it is easy to check that $m$ is a core point of $N_{\tau}$ and $N^{\infty} \subset C(T)$. If $A \neq \mathscr{A}$, then $H^{\infty}$ is contained properly in the classical Hardy space. Hence $H^{\infty}$ is not $\tau$-maximal. On the other hand if $\tau$ has a unique logmodular measure $m$, then $H^{\infty}$ is $\tau$-maximal ([9, Theorem 5.5]). This implies that $m$ is not a unique logmodular measure and hence Theorem 2 implies that $\gamma_{0}$ is infinite.

(IV) The unit polydisc $U^{n}$ and the torus $T^{n}$ are cartesian products of $n$ copies of the unit disc $U$ and of the unit circle $T$, respectively. $A\left(U^{n}\right)$ is the class of all continuous complex functions on the closure $\bar{U}^{n}$ of $U^{n}$ with holomorphic restrictions to $U^{n}$. Let $A=A\left(U^{n}\right) \mid X$ and $X=T^{n}$. This is the so-called polydisc algebra. For simplicity we assume $n=2$. Let $m$ be the normalized Lebesgue measure; then $m$ is a representing measure for $\tau$ on $X$ where $\tau(f)=f(0)$ and $0 \in U^{2}$. Suppose $1 \leqq p \leqq \infty$ and $Z_{+}^{2}=$ $\left\{(n, m) \in \boldsymbol{Z}^{2} ; n \geqq 0\right.$ and $\left.m \geqq 0\right\}$. Then $H^{p}=\left\{f \in L^{p} ; \hat{f}(n, m)=0\right.$ if $\left.(n, m) \notin Z_{+}^{2}\right\}$ and $K^{p}=\left\{f \in L^{p} ; \hat{f}(n, m)=0\right.$ if $\left.(-n,-m) \in Z_{+}^{2}\right\} . K^{\infty}$ is dense in $K^{p}$. Unfortunately we do not know whether $\gamma_{0}$ is finite or not.

3. Norms of Hankel operators. Assuming $\gamma_{0}$ is finite, we show that $\left\|H_{\phi}^{(1)}\right\|$ (resp. $\left.\left\|H_{\phi}^{(2)}\right\|\right)$ is equivalent to $\left\|\phi+H^{\infty}\right\|$ (resp. $\left.\left\|\phi+K^{\infty}\right\|\right)$.

Theorem 3. Let $\phi$ be a function in $L^{\infty}$. Then

$$
\left\|H_{\phi}^{(2)}\right\| \leqq\left\|\phi+K^{\infty}\right\| \leqq \gamma_{0}\left\|H_{\phi}^{(2)}\right\| \text {. }
$$

If $K^{\infty}$ is dense in $K^{1}$, then

$$
\left\|H_{\phi}^{(1)}\right\| \leqq\left\|\phi+H^{\infty}\right\| \leqq \gamma_{0}\left\|H_{\phi}^{(1)}\right\| \text {. }
$$

Proof. Let $v \in\left(L^{\infty}\right)_{+}^{-1}$. If $\int_{X}|f|^{2} v^{2} d m \leqq 1$ and $\int_{X}|g|^{2} v^{-2} d m \leqq 1$, then $\int_{X}|f|^{2} d m \leqq\left\|v^{-2}\right\|_{\infty}$ and $\int_{X}|g|^{2} d m \leqq\left\|v^{2}\right\|_{\infty}$. Hence

$$
\begin{aligned}
\left\|H_{\phi}^{(1) v}\right\| & =\sup \left\{\left|\int_{X} f g \phi d m\right| ; f \in H^{2}, g \in K_{0}^{2}, \int_{X}|f|^{2} v^{2} d m \leqq 1 \text { and } \int_{X}|g|^{2} v^{-2} d m \leqq 1\right\} \\
& \leqq\left(\left\|v^{-2}\right\|_{\infty}\left\|v^{2}\right\|_{\infty}\right)^{1 / 2}\left\|H_{\phi}^{(1)}\right\| .
\end{aligned}
$$

If $h \in\left(H^{\infty}\right)^{-1}$, then $v|h| H^{2}=b\left(v H^{2}\right)$ and $v^{-1}|h|^{-1} \bar{K}_{0}^{2}=b\left(v^{-1} \bar{K}_{0}^{2}\right)$ with $b=|h| / h$. Then $Q_{v|h|}^{(1)}=M_{b} Q_{v}^{(1)} M_{\bar{b}}$ and so $H_{\phi}^{(1) v|h|}=M_{b} Q_{v}^{(1)} M_{\bar{b}} M_{\phi}|v| h \mid H^{2}$. Hence $\left\|H_{\phi}^{(1) v}\right\|=$ $\left\|H_{\phi}^{(1) v|h|}\right\|$. Thus for any $h \in\left(H^{\infty}\right)^{-1}$

$$
\left\|H_{\phi}^{(1) v}\right\| \leqq\|v|h|\|_{\infty}\left\|v^{-1}|h|^{-1}\right\|_{\infty}\left\|H_{\phi}^{(1)}\right\|
$$


It is easy to see that

$$
\sup _{v \in\left(L^{\infty}\right)-1}\left\{\inf \left\{\|v|h|\|_{\infty}\left\|v^{-1}|h|^{-1}\right\|_{\infty} ; h \in\left(H^{\infty}\right)^{-1}\right\}\right\}=\gamma_{0} .
$$

Now generalized Nehari's Theorem I shows that $\left\|H_{\phi}^{(1)}\right\| \leqq\left\|\phi+H^{\infty}\right\| \leqq$ $\gamma_{0}\left\|H_{\phi}^{(1)}\right\|$. Similarly the inequality for $H_{\phi}^{(2)}$ follows.

4. Applications. In the previous paper [14] we gave applications of generalized Nehari's Theorems I and II to weighted norm inequalities and invertible Toeplitz operators. In this section we shall give applications of Theorem 3.

Recall $P$ is the orthogonal projection from $L^{2}$ to $H^{2}$. Let $\mathscr{P}^{(1)}$ denote $P$ restricted to $H^{\infty}+\bar{K}_{0}^{\infty}$ and $\mathscr{P}^{(2)}$ denote $P$ restricted to $H^{\infty}+\bar{H}_{0}^{\infty}$. We are interested in knowing when $\mathscr{P}^{(j)}(j=1,2)$ is bounded in $L^{2}(w)=$ $L^{2}(w d m)$, where $w$ is a nonnegative weight function in $L^{1}$. Put

(d) $\sup \left\{\left|\int_{X} f g w d m\right| ; f \in H^{\infty}, g \in K_{0}^{\infty}\right.$ and $\left.\int_{X}|f|^{2} w d m=\int_{X}|g|^{2} w d m=1\right\}$ and

$$
=\rho_{1}
$$

(e) $\sup \left\{\left|\int_{X} f g w d m\right| ; f \in H^{\infty}, g \in H_{0}^{\infty}\right.$ and $\left.\int_{X}|f|^{2} w d m=\int_{X}|g|^{2} w d m=1\right\}$

$$
=\rho_{2} \text {. }
$$

Then it is easy to see that $\left\|\mathscr{P}^{(j)}\right\|^{2} \leqq\left(1-\rho_{j}^{2}\right)^{-1}$. The following lemma is known [19]. We shall give the proof for completeness.

LEMMA 3. $\left\|\mathscr{P}^{(j)}\right\|^{2}=\left(1-\rho_{j}^{2}\right)^{-1}(j=1,2)$.

Proof. If $\gamma=\left\|\mathscr{P}^{(1)}\right\|<\infty$, then for any real $t$ and for any $f \in H^{\infty}$ and $g \in K_{0}^{\infty}$ we have

$$
t^{2} \frac{\gamma^{2}-1}{\gamma^{2}} \int_{X}|f|^{2} w d m+\int_{X}|g|^{2} w d m+2 t \operatorname{Re} \int_{X} f g w d m \geqq 0 .
$$

Hence

$$
\left|\int_{X} f g w d m\right|^{2} \leqq \frac{\gamma^{2}-1}{\gamma^{2}} \int_{X}|f|^{2} w d m \int_{X}|g|^{2} w d m
$$

and so $\gamma^{2} \geqq\left(1-\rho_{1}^{2}\right)^{-1}$. We can prove it for $j=2$ in the same method.

If $A$ is a disc algebra, then $\mathscr{P}=\mathscr{P}^{(1)}=\mathscr{P}^{(2)}$ is bounded in $L^{2}(w)$ if and only if $w=|h|^{2}$ for some outer function $h$ in $H^{2}$ and $\left\|\phi+H^{\infty}\right\|<1$ with $\phi=|h|^{2} / h^{2}$. This result is called Helson-Szegö's theorem [12]. This was generalized to general uniform algebras by the author [14]. The following generalization seems to be better than the previous one.

COROLLARY 1. Suppose $K^{\infty}$ is dense in $K^{1}$. Let $w=|h|^{2}$ for some function $h$ in $H^{2}$ such that $h H^{\infty}$ is dense in $H^{2}$ and $h K^{\infty}$ is dense in $K^{2}$. 
Let $\phi=|h|^{2} / h^{2}$.

(1) If $\left\|\phi+H^{\infty}\right\|=\rho<1$ then $\mathscr{P}^{(1)}$ is bounded in $L^{2}(w)$ and $\left\|\mathscr{P}^{(1)}\right\| \leqq$ $\left(1-\rho^{2}\right)^{-1 / 2}$.

(2) If $\mathscr{P}^{(1)}$ is bounded in $L^{2}(w)$ and $\left\|\mathscr{P}^{(1)}\right\|=\gamma$ then

$$
\left\|\dot{\phi}+H^{\infty}\right\|<\gamma_{0} \gamma^{-1}\left(\gamma^{2}-1\right)^{1 / 2}
$$

Hence if $\gamma<\gamma_{0}\left(\gamma_{0}^{2}-1\right)^{1 / 2}$ then $\left\|\phi+H^{\infty}\right\|<1$.

PROoF. (1) $\rho_{1} \leqq \rho$, since

$$
\begin{aligned}
\rho & =\left\|\phi+H^{\infty}\right\|=\sup \left\{\left|\int_{X} F \phi d m\right| ; F \in K_{0}^{1} \text { and }\|F\|_{1} \leqq 1\right\} \\
& \geqq \sup \left\{\left|\int_{X} \phi f g d m\right| ; f \in H^{2}, g \in K_{0}^{2} \text { and } \int_{X}|f|^{2} d m=\int_{X}|g|^{2} d m=1\right\}=\rho_{1} .
\end{aligned}
$$

In the last equality we used the facts that $w=|h|^{2}$ and that $h H^{\infty}$ (resp. $\left.h K_{0}^{\infty}\right)$ is dense in $H^{2}$ (resp. $K_{0}^{2}$ ).

(2) Since $\rho_{1}=\gamma^{-1}\left(\gamma^{2}-1\right)^{1 / 2}$ by Lemma 3 and $\rho_{1}=\left\|H_{\phi}^{(1)}\right\|$ by the proof of (1), Theorem 3 implies (2).

$K^{\infty}$ is dense in $K^{2}$ if we impose the assumptions (1) and (2) or if $A$ is a polydisc algebra. We have a similar result for $\mathscr{P}^{(2)}$ (or $\left.\left\|\phi+K^{\infty}\right\|\right)$ as in Corollary 1.

For $\phi$ in $L^{\infty}$ let $T_{\phi}$ be the operator on $H^{2}$ defined by $T_{\phi} f=P\left(M_{\phi} f\right)$. The operator $T_{\phi}$ will be called a Toeplitz operator. We are interested in knowing when $T_{\phi}$ is left invertible. In case $A$ is a disc algebra, Widom [18] showed that $T_{\phi}$ is left invertible if and only if $\left\|\phi+H^{\infty}\right\| .<1$. Abrahamse [1] generalized Widom's theorem to the case of (I) in concrete uniform algebras such that $\partial Y$ consists of $n+1$ non-intersecting analytic Jordan curves. The author [14] generalized it to general uniform algebras. However these generalizations are not sufficient because except in the case of a disc algebra we cannot determine $\phi$ when $T_{\phi}$ is left invertible.

COROLlary 2. Suppose $K^{\infty}$ is dense in $K^{1}$. Let $\phi$ be a unimodular function in $L^{\infty}$. in $H^{2}$.

(1) If $\left\|\phi+H^{\infty}\right\|=\rho<1$, then $\left\|T_{\phi} f\right\|_{2} \geqq\left(1-\rho^{2}\right)^{1 / 2}\|f\|_{2}$ for any $f$

(2) If $\left\|T_{\phi} f\right\|_{2} \geqq \varepsilon\|f\|_{2}$ for any $f$ in $H^{2}$, then

$$
\left\|\phi+H^{\infty}\right\| \leqq \gamma_{0}\left(1-\varepsilon^{2}\right)^{1 / 2} .
$$

Hence if $\varepsilon>\gamma_{0}^{-1}\left(\gamma_{0}^{2}-1\right)^{1 / 2}$, then $\left\|\phi+H^{\infty}\right\|<1$.

Proof. Since $\phi$ is a unimodular function, $\left\|H_{\phi}^{(1)} f\right\|_{2}^{2}+\left\|T_{\phi} f\right\|_{2}^{2}=\|f\|_{2}^{2}$ for any $f \in H^{2}$. Theorem 3 and this imply the corollary.

In the case of (I) for concrete uniform algebras, $\left\|\phi+H^{\infty}\right\|<1$ may 
not hold even if $T_{\phi}$ is left invertible (cf. [1]).

5. Essential norms of Hankel operators. In this section we shall concentrate on concrete uniform algebras, that is, (I) in Section 2 such that $\partial Y$ consists of $n+1$ non-intersecting analytic Jordan curves. Hence $\tau$ satisfies the conditions (1) and (3). Using generalized Nehari's Theorem II we shall generalize (b) in Introduction to this context.

Let $s=\left(s_{1}, s_{2}, \cdots s_{n}\right) \in I^{n}=[0,1] \times \cdots \times[0,1]$. Then the mapping $s \mapsto$ $\exp \left(\sum_{j=1}^{n} s_{j} u_{j}\right)$ is continuous, one-to-one and onto from $I^{n}$ to $\mathscr{E}_{0}$. Put

$$
H_{\phi}^{(j) s}=H_{\phi}^{(j) v} \quad(j=1,2)
$$

where $v=\exp \left(\sum_{j=1}^{n} s_{j} u_{j}\right)$.

LEMMA 4. Let $\phi$ be a function in $L^{\infty}$. Then for $j=1,2$ and for any $v$ and $u$ in $\mathscr{E}_{0}$

$$
\left\|H_{\phi}^{(j) v}-H_{\phi}^{(j) u}\right\| \leqq\|\phi\|_{\infty}\left(2 \sup _{v \in \mathscr{E}_{0}}\left\|v^{-1}\right\|_{\infty}\right)\|v-u\|_{\infty} .
$$

The proof is clear by Lemma 1.

LEMmA 5. If $\phi$ in $H^{\infty}+C(X)$, then $H_{\phi}^{(j) v}(j=1,2)$ is compact for any $v$ in $\mathscr{E}_{0}$.

Proof. By Lemma 2 it is sufficient to show that $H_{\phi}^{(j)}$ is compact for any $\phi \in C(X)$. Let $\phi=(z-a)^{-1}$ for some $a \in Y^{0}$. Then

$$
H_{\phi}^{(j)} f=Q^{(j)}\left[\frac{f(a)}{z-a}+\frac{f-f(a)}{z-a}\right]=Q^{(j)}\left[\frac{f(a)}{z-a}\right]
$$

for any $f \in H^{2}$ because $\left\{f \in H^{2}: f(a)=0\right\}=(z-a) H^{2}$. Hence $H_{\phi}^{(j)}$ has rank one. Similarly if $\phi=(z-a)^{-n}$ for a positive integer $n$, we can show that $H_{\phi}^{(j)}$ has rank $n$. For any $\phi \in C(X)$ we can approximate $\phi$ by the following functions: $\sum_{j=0}^{n} b_{j}\left(z-a_{j}\right)^{-j}$ where $a_{j} \in Y^{0}$ and $b_{j}$ is constant $(0 \leqq j \leqq n)$. Since $\left\|H_{\phi}^{(1)}\right\| \leqq\left\|\phi+H^{\infty}\right\|$ and $\left\|H_{\phi}^{(2)}\right\| \leqq\left\|\phi+H^{\infty}+N_{c}^{\infty}\right\|$, we can show that $H_{\phi}^{(j)}$ is compact if $\phi \in C(X)$.

THEOREM 4. Let $\phi$ be a function in $L^{\infty}$. Then

$$
\sup _{v \in \mathbb{8}_{0}}\left\|H_{\phi}^{(1) v}\right\|_{e}=\sup _{v \in \mathbb{8}_{0}}\left\|H_{\phi}^{(2) v}\right\|_{\delta}=\left\|\phi+H^{\infty}+C(X)\right\| \text {. }
$$

Moreover, the suprema in both equalities are attained.

Proof. By Lemma 5 it is clear that $\sup \left\{\left\|H_{\phi}^{(j) v}\right\|_{e} ; v \in \mathscr{E}_{0}\right\} \leqq \| \phi+H^{\infty}+$ $C(X) \|$ for $j=1,2$. We shall show the opposite inequality. Let $F$ be the Ahlfors function for $Y^{0}$ and $\tau \in Y^{0}$. Then $F \in C(X)$ (see [8, p. 114]). For any $v \in \mathscr{E}_{0}$ with $v=\exp \left(\sum_{j=1}^{n} t_{j} u_{j}\right)$ and $t=\left(t_{1}, t_{2}, \cdots, t_{n}\right) \in I^{n}$, put

$$
f^{(j)}(t, l)=\left\|H_{F l_{\phi}}^{(j) t}\right\| \quad(l=0,1,2, \cdots ; j=1,2) \text {. }
$$


Then $f^{(j)}(t, l) \geqq f^{(j)}(t, l+1)$ and by Lemma 4

$$
\left|f^{(j)}(t, l)-f^{(j)}(s, l)\right| \leqq\|\phi\|_{\infty}\left(2 \sup _{v \in \mathbb{E}_{0}}\left\|v^{-1}\right\|_{\infty}\right)\left\|\exp \left(\sum_{j=1}^{n} t_{j} n_{j}\right)-\exp \left(\sum_{j=1}^{n} s_{j} u_{j}\right)\right\|_{\infty} .
$$

Hence $\left\{f^{(j)}(t, l)\right\}_{l=1}^{\infty}$ is an equicontinuous collection on $I^{n}$ and uniformly bounded on $I^{n}$. By Ascoli's theorem, there exists a subsequence $\left\{f^{(j)}\left(t, l_{i}\right)\right\}_{i=1}^{\infty}$ of $\left\{f^{(j)}(t, l)\right\}_{l=1}^{\infty}$ and a continuous function $f^{(j)}(t)$ on $I^{n}$ such that

$$
\sup _{t \in I^{n}}\left|f^{(j)}(t)-f^{(j)}\left(t, l_{i}\right)\right| \rightarrow 0 \quad(\text { as } i \rightarrow \infty) .
$$

Since $\left\{f^{(j)}(t, l)\right\}_{l=1}^{\infty}$ is a decreasing sequence, this actually converges to $f^{(j)}(t)$ uniformly on $I^{n}$. Thus

$$
\lim _{l} \sup _{t \in I^{n}} f^{(j)}(t, l)=\sup _{t \in I^{n}} f^{(j)}(t) \text {. }
$$

By generalized Nehari's Theorem II, $\sup \left\{f^{(1)}(t, l) ; t \in I^{n}\right\}=\left\|F^{l} \phi+H^{\infty}\right\|$ and $\sup \left\{f^{(2)}(t, l) ; t \in I^{n}\right\}=\left\|F^{l} \phi+H^{\infty}+N_{c}^{\infty}\right\|$ and so for $j=1,2$

$$
\sup _{t \in I^{n}} f^{(j)}(t) \geqq\left\|\phi+H^{\infty}+C(X)\right\| \text {, }
$$

because the closure of $\cup_{n=1}^{\infty} \bar{F}^{n} H^{\infty}$ coincides with the closure of $\cup_{n=1}^{\infty} \bar{F}^{n}\left(H^{\infty}+\right.$ $N_{c}^{\infty}$ ), which is $H^{\infty}+C(X)$ (cf. [1, Theorem 1.22]). For any $t \in I^{n}$, let $S_{t}$ denote the multiplication by $F$ on $v H^{2}$ where $v=\exp \left(\sum_{j=1}^{n} t_{j} u_{j}\right)$. Let $S_{t}^{(1) *}$ be the adjoint of $S_{t}$ from $v H^{2}$ to $v^{-1} \bar{K}_{0}^{2}$ and $S_{t}^{(2) *}$ the adjoint of $S_{t}$ from $v H^{2}$ to $v^{-1} \bar{H}_{0}^{2}$. If $K_{t}^{(1)}$ is any compact operator from $v H^{2}$ to $v^{-1} \bar{K}_{0}^{2}$ and $K_{t}^{(2)}$ is any compact operator from $v H^{2}$ to $v^{-1} \bar{H}_{0}^{2}$, and $l$ is positive integer, then for $j=1,2$

$$
\left\|H_{\phi}^{(j) t}-K_{t}^{(j)}\right\| \geqq\left\|\left(H_{\phi}^{(j) t}-K_{t}^{(j)}\right) S_{t}^{l}\right\| \geqq\left\|H_{\phi}^{(j) t} S_{t}^{l}\right\|-\left\|K_{t}^{(j)} S_{t}^{l}\right\| .
$$

Since $\left(S_{t}^{(j) l}\right)^{*} \rightarrow 0$ strongly, we have $\left\|K_{t}^{(j)} S_{t}^{l}\right\| \rightarrow 0$. Also

$$
H_{\phi}^{(j) t} S_{t}^{l}=H_{F l_{\phi}}^{(j) t} \text {. }
$$

Hence we can prove that the suprema are attained as in the proof of generalized Nehari's Theorem II.

$$
\left\|H_{\phi}^{(j) t}-K_{t}^{(j)}\right\| \geqq \varlimsup
$$

Thus $\left\|H_{\phi}^{(j) t}\right\|_{e} \geqq f^{(j)}(t)$ and

$$
\sup _{t \in I^{n}}\left\|H_{\phi}^{(j) t}\right\|_{e} \geqq \sup _{t \in I^{n}} f^{(j)}(t) \geqq\left\|\phi+H^{\infty}+C(X)\right\| \text {. }
$$

The following theorem is another generalization of (b) in Introduction.

THEOREM 5. Let $\phi$ be a function in $L^{\infty}$. Then for $j=1,2$

$$
\left\|H_{\phi}^{(j)}\right\|_{e} \leqq\left\|\phi+H^{\infty}+C(X)\right\| \leqq \gamma_{0}\left\|H_{\phi}^{(j)}\right\|_{e} \text {. }
$$


The proof follows as in the case of a disc algebra (see [16, Theorem 1.4]) if we use Theorem 3 and the Ahlfors function.

6. Factorization theorems. We say $H_{0}^{1}$ has the weak approximate $\gamma$-factorization if $H_{0}^{1}$ satisfies the following property: For any $F$ in $H_{0}^{1}$ and any $\varepsilon>0$, there exist $\left\{f_{j}\right\}_{j=1}^{n}$ in $H^{2}$ and $\left\{g_{j}\right\}_{j=1}^{n}$ in $H_{0}^{2}$ such that

and

$$
\sum_{j=1}^{n}\left\|f_{j}\right\|_{2}\left\|g_{j}\right\|_{2} \leqq \gamma\|F\|_{1}
$$

$$
\left\|F-\sum_{j=1}^{n} f_{j} g_{j}\right\|_{1}<\varepsilon .
$$

Proposition 6. There exists a constant $\gamma$ with $\gamma \geqq 1$ such that $\left\|\phi+K^{\infty}\right\| \leqq \gamma\left\|H_{\phi}^{(2)}\right\|$ for all $\phi$ in $L^{\infty}$ if and only if $H_{0}^{1}$ has the weak approximate $\gamma$-factorization.

Proof. Let $V_{\gamma}$ be the closure in $L^{1}$ of the following set:

$$
\left\{\sum_{j=1}^{n} f_{j} g_{j} ; f_{j} \in H^{2}, g_{j} \in H_{0}^{2} \text { and } \sum_{j=1}^{n}\left\|f_{j}\right\|_{2}\left\|g_{j}\right\|_{2} \leqq \gamma\right\} \text {. }
$$

Put $V^{1}=\left\{F \in H_{0}^{1} ;\|F\|_{1} \leqq 1\right\}$. Then $V_{\gamma}$ is the closed convex subset in $\gamma V^{1}$. If $H_{0}^{1}$ has the weak approximate $\gamma$-factorization, then $V^{1} \subset V_{r}$ and so $\left\|\phi+K^{\infty}\right\| \leqq \gamma\left\|H_{\phi}^{(2)}\right\|$, since

$$
\left|\int_{X}\left(\sum_{j=1}^{n} f_{j} g_{j}\right) \phi d m\right| \leqq\left\|H_{\phi}^{(2)}\right\| \sum_{j=1}^{n}\left\|f_{j}\right\|_{2}\left\|g_{j}\right\|_{2} \text {. }
$$

Conversely, suppose $\left\|\phi+K^{\infty}\right\| \leqq \gamma\left\|H_{\phi}^{(2)}\right\|$. If $H_{0}^{1}$ does not have the weak approximate $\gamma$-factorization, then there exists $F \in V^{1}$ with $F \notin V_{r}$. Then by the Hahn-Banach theorem there exists $\phi \in L^{\infty}$ such that

$$
\left|\int_{X} \phi F d m\right|>\sup \left\{\left|\int_{X} \phi f d m\right| ; f \in V_{r}\right\}
$$

and so $\left\|\phi+K^{\infty}\right\|>\gamma\left\|H_{\phi}^{(2)}\right\|$.

For $K_{0}^{1}$ we can define the weak approximate $\gamma$-factorization and prove Proposition 6 with $H_{\phi}^{(1)}, H^{\infty}$ and $K_{0}^{1}$ instead of $H_{\phi}^{(2)}, K^{\infty}$ and $H_{0}^{1}$, respectively. In (I) for concrete uniform algebras we have factorization theorems of $H_{0}^{1}$ and $K_{0}^{1}$. M. Hayashi pointed out a factorization theorem of $H_{0}^{1}$. We now give a proof and clarify its relationship with $\gamma_{0}$.

THEOREM 7. Suppose $A$ is a concrete uniform algebra (I).

(1) If $f$ is in $H_{0}^{1}$, then there is a $g$ in $H^{2}$ and an $h$ in $H_{0}^{2}$ such that $f=g h$ and $\|g\|_{2}\|h\|_{2} \leqq \gamma_{2}\|f\|_{1}$, where $\gamma_{2}=\sup \left\{\left\|v^{-1}\right\|_{\infty}\|v\|_{\infty} ; v \in \mathscr{E}_{0}\right\}$. In this case $\gamma_{2} \geqq \gamma_{0}$.

(2) If $f_{1}$ is in $K_{0}^{1}$, then there is a $g_{1}$ in $H^{2}$ and an $h_{1}$ in $K_{0}^{2}$ such 
that $f_{1}=g_{1} h_{1}$ and $\left\|g_{1}\right\|_{2}\left\|h_{1}\right\|_{2} \leqq \gamma_{3}\left\|f_{1}\right\|_{1}$, where $\gamma_{3}=\gamma_{2}\left\|v_{0}\right\|_{\infty}$ and $K_{0}^{1}=v_{0} H_{0}^{1}$.

Proof. (1) A function $f \in H_{0}^{1}$ is of the form $f=F G^{2}$ where $F \in H_{0}^{\infty}$ with $|F| \in \mathscr{E}$ and $G \in H^{2}$ [3, p. 138]. If $|F|=\exp \left(\sum_{j=1}^{n} t_{j} u_{j}\right)$, let $k=\prod_{j=1}^{n}\left(h_{j}\right)^{l_{j}}$ and $l_{j}=\left[t_{j} / 2\right]$. Then $k \in\left(H^{\infty}\right)^{-1}$. Put $s_{j}=2\left(t_{j} / 2-\left[t_{j} / 2\right]\right)$. Then $q=F k^{-1} \in$ ${ }^{i} H_{0}^{\infty}$ and $|q|=\exp \left(\sum_{j=1}^{n} s_{j} u_{j}\right) \in \mathscr{E}_{0}^{2}=\left\{v^{2} ; v \in \mathscr{E}_{0}\right\}$. Let $g=k G$ and $h=q G$. Then $f=g h$ and

$$
\begin{aligned}
\int_{X}|g|^{2} d m \int_{X}|h|^{2} d m & =\int_{X}\left|q^{-1}\right||f| d m \int_{X}|q||f| d m \leqq\left\|q^{-1}\right\|_{\infty}\|q\|_{\infty}\left[\int_{X}|f| d m\right]^{2} \\
& \leqq \sup _{X}\left\{\left\|q^{-1}\right\|_{\infty}\|q\|_{\infty} ;|q| \in \mathscr{E}_{0}^{2}\right\}\left[\int_{X}|f| d m\right]^{2} .
\end{aligned}
$$

If $u \in\left(L^{\infty}\right)_{+}^{-1}$ then $u=v|g|$ with $v \in \mathscr{E}_{0}$ and $g \in\left(H^{\infty}\right)^{-1}$. Hence

$$
\gamma_{2}=\sup \left\{\|v\|_{\infty}\left\|v^{-1}\right\|_{\infty} ; v \in \mathscr{E}_{0}\right\} \geqq \sup \left\{\|(u)\| ; u \in\left(L^{\infty}\right)_{+}^{-1}\right\}=\gamma_{0} .
$$

(2) A function $f_{1} \in K_{0}^{1}$ is of the form $f_{1}=v_{0} f$ for some $f \in H_{0}^{1}$. Apply (1) to this $f$, and let $g_{1}=g$ and $h_{1}=v_{0} h$, then $g_{1} \in H^{2}$ and $h_{1} \in K_{0}^{2}$. Now (2) follows.

(1) of Theorem 7 gives $\left\|H_{\phi}^{(2)}\right\| \leqq\left\|\phi+K^{\infty}\right\| \leqq \gamma_{2}\left\|H_{\phi}^{(2)}\right\|$ in the case of (I) for concrete uniform algebras.

(2) of Theorem 7 gives that $\left\|H_{\phi}^{(1)}\right\| \leqq\left\|\phi+H^{\infty}\right\| \leqq \gamma_{s}\left\|H_{\phi}^{(1)}\right\|$. For any uniform algebra with finite $\gamma_{0}$, Theorem 3 and Proposition 6 show that both $H_{0}^{1}$ and $K_{0}^{1}$ have the weak approximate $\gamma_{0}$-factorizations.

7. Arveson's distance formula. Let $\mathscr{A}$ be a (possibly non-selfadjoint) algebra of operators on a Hilbert space $\mathscr{H}$, and let $T$ be an arbitrary bounded operator. Then $d(T, \mathscr{A}) \geqq \sup _{P}\|(1-P) T P\|$, where $d(T, \mathscr{A})$ is the distance from $T$ to $\mathscr{A}$ and where the supremum is taken over the lattice lat $\mathscr{A}$ of all $\mathscr{A}$-invariant projections. Arveson [5, Theorem 1.1.] showed that if $\mathscr{A}$ is a nest algebra (i.e., lat $\mathscr{A}$ is totally ordered) then the equality holds above. Let $\mathscr{H}=L^{2}$ and $P_{v}^{(1)}=1-Q_{v}^{(1)}$. Generalized Nehari's Theorem I implies that if $K^{\infty}$ is dense in $K^{1}$ and lat $\mathscr{A} \ni P_{v}^{(1)}$ for any $v$ in $\left(L^{\infty}\right)_{+}^{-1}$, then for any $\phi$ in $L^{\infty}$

$$
d\left(M_{\phi}, \mathscr{A}\right)=\sup _{P}\left\|(I-P) M_{\phi} P\right\| .
$$

Let $\mathscr{C}(\mathscr{H})$ be the space of all compact operators on $\mathscr{H}$ and $\mathscr{B}$ the the norm closure of $\mathscr{A}+\mathscr{C}(\mathscr{C})$. Then $d(T, \mathscr{B}) \geqq \sup _{P}\|(I-P) T P\|_{e}$. Theorem 4 implies that if lat $\mathscr{A} \ni P_{v}^{(1)}$ for any $v$ in $\mathscr{E}_{0}$ then

$$
d\left(M_{\phi}, \mathscr{B}\right)=\sup _{P}\left\|(I-P) M_{\phi} P\right\|_{\text {. }} \text { for any } \phi \text { in } L^{\infty} .
$$




\section{REFERENCES}

[1] M. B. Abrahamse, Toeplitz operators in multiply connected regions, Amer. J. Math. 96 (1974), 261-297.

[2] V. M. Adamuan, D.Z. Arov and M. G. Krein, Analytic properties of Schmidt pairs for a Hankel operator and the generalized Schur-Takagi problem, Matem. Sbornik 86 (128) (1971), 34-35; English translation: Math. USSR Sbornik 15 (1971), 31-73.

[3] P. Ahern and D. Sarason, The $H^{P}$ spaces of a class of function algebras, Acta Math. 117 (1967), 123-163.

[4] J.M. ANDERSON AND R. H. ROCHBERG, Toeplitz operators associated with subalgebras of the disc algebra, Indiana Univ. Math. J. 30 (1981), 813-820.

[5] W. B. ARveson, Interpolation problems in nest algebras, J. Functional Analysis 20 (1975), 208-233.

[6] R. E. Curto, P.S. Muhly, T. NAKazi and J. XIA, Hankel operators and uhiform algebras, Archiv der Math. 43 (1984), 440-447.

[7] R. G. Douglas and C. Pearcy, On a topology for invariant subspaces, J. Functional Analysis 2 (1968), 323-341.

[8] S.D. FIsher, Function Theory on Planar Domains, A Wiley-Interscience Publication.

[9] T. GAMELIN, Embedding Riemann surfaces in maximal ideal spaces, J. Functional Analysis 2 (1968), 123-146.

[10] T. Gamelin, Uniform Algebras, Prentice-Hall, Englewood Cliffs, New Jersey, 1969.

[11] P. Hartman, On completely continuous Hankel matrices, Proc. Amer. Math. Soc. 9 (1958), 862-866.

[12] H. Helson AND G. Szegö, A problem in prediction theory, Ann. Math. Pura Appl. 51 (1960), 107-138.

[13] K. Hoffman, Banach Spaces of Analytic Functions, Prentice-Hall, Englewood Cliffs, New Jersey, 1962.

[14] T. NakazI, Norms of Hankel operators and uniform algebras, Trans. Amer. Math. Soc. 299 (1987), 573-580.

[15] Z. NeHARI, On bounded bilinear forms, Ann. of Math. 65 (1957), 153-162.

[16] Y. OHNO, Remarks on Helson-Szegö problems, Tôhoku Math. J. 18 (1965), 54-59.

[17] S. C. Power, Hankel Operators on Hilbert Space, Research Notes in Math. 64, Pitman Advanced Publishing Program, 1982.

[18] R. ROCHBERG, Function algebra invariants and the conformal structure of planar domains. J. Functional Analysis 13 (1973), 154-172.

[19] T. Yамамото, Weighted norm inequalities in Hilbert space $L^{2}$, master's thesis, Hokkaido University 1983.

Department of Mathematics

Faculty of Science (General Education)

HOKKAIDO UNIVERSITY

SAPPORO 060

JAPAN 
